Revue internationale P.M.E.

Économie et gestion de la petite et moyenne entreprise

\title{
Incidence de la loi proxémique sur la perception de l'incertitude des PME
}

\section{Olivier Torrès et Gaël Gueguen}

Volume 21, numéro 1, 2008

URI : https://id.erudit.org/iderudit/038004ar

DOI : https://doi.org/10.7202/038004ar

Aller au sommaire du numéro

Éditeur(s)

Presses de l'Université du Québec

ISSN

0776-5436 (imprimé)

1918-9699 (numérique)

Découvrir la revue

Citer cet article

Torrès, O. \& Gueguen, G. (2008). Incidence de la loi proxémique sur la perception de l'incertitude des PME. Revue internationale P.M.E., 21(1), 93-117. https://doi.org/10.7202/038004ar

\section{Résumé de l'article}

Les travaux concernant les PME suggèrent une spécificité induisant des modalités de gestion particulières des entreprises de petite dimension. Toutefois, sur quels ressorts repose cette spécificité ? La thèse défendue sera que la spécificité de la gestion des PME est la proximité. De nombreuses études ont démontré la préférence des PME, par l'intermédiaire de leur propriétaire-dirigeant, pour des relations de proximité. Nous chercherons à affiner cette vision en essayant de voir l'impact de cette proximité en ce qui concerne la perception d'incertitude de la part des PME. Sur la base d'une étude quantitative menée auprès de 239 PME et de tests statistiques à dimension exploratoire, nous essayerons d'appréhender deux propositions de recherche : la proximité est un réducteur d'incertitude et la proximité est un facteur de performance. Les résultats obtenus nous conduiront à envisager l'importance des relations de proximité. 


\title{
Incidence de la loi proxémique sur la perception de l'incertitude des PME
}

\author{
Olivier TORRÈS \\ EM Lyon, Université de Montpellier III \\ Gaël GUEGUEN \\ Université de Montpellier III
}

MOTS CLÉS

PME - Proximité - Loi proxémique - Incertitude

\begin{abstract}
LES AUTEURS
OLIVIER ToRRĖs est maître de conférences habilité en sciences de gestion à l'Université de Montpellier (ERFI) et chercheur associé à l'EM Lyon. Il est secrétaire de l'Association internationale de recherche en entrepreneuriat et en PME (AIREPME) et vice-président pour la France de l'European Council for Small Business (ECSB). Ses travaux portent principalement sur les fondements de la spécificité de management des entreprises de petite taille (PME et TPE). Adresse: Université de Montpellier III, route de Mende, 34199 Montpellier, Cédex 5, France. Courriel : <olivier.torres@univ-montp3.fr>.

GAËL GUEGUEN est maître de conférences en sciences de gestion à l'Université de Montpellier III et appartient à l'Équipe de recherche sur la firme et l'industrie (ERFI) de l'Université de Montpellier I. II a soutenu en 2001 sa thèse de doctorat sur la thématique du management stratégique des PME et l'importance de leur environnement. Trésorier de l'Association internationale de recherche en entrepreneuriat et en PME (AIREPME), ses recherches portent également sur les mouvements stratégiques des entreprises au sein des technologies de l'information. Adresse: Université de Montpellier III, route de Mende, 34199 Montpellier, Cédex 5, France. Courriel:<gael.gueguen@univ-montp3.fr>.
\end{abstract}

\section{RÉSUMÉ}

Les travaux concernant les PME suggèrent une spécificité induisant des modalités de gestion particulières des entreprises de petite dimension. Toutefois, sur quels ressorts repose cette spécificité ? La thèse défendue sera que la spécificité de la gestion des PME est la proximité. De nombreuses études ont démontré la préférence des PME, par l'intermédiaire de leur propriétaire-dirigeant, pour des relations de proximité. Nous chercherons à affiner cette vision en essayant de voir l'impact de cette proximité en ce qui concerne la perception d'incertitude de la part des PME. Sur la base d'une étude quantitative menée auprès de 239 PME et de 
tests statistiques à dimension exploratoire, nous essayerons d'appréhender deux propositions de recherche: la proximité est un réducteur d'incertitude et la proximité est un facteur de performance. Les résultats obtenus nous conduiront à envisager l'importance des relations de proximité.

\begin{abstract}
Researches on SMEs underline the specificities of these structures leading to a particular management style. Nevertheless, how to define this specificity? The thesis defended in this contribution is the following: SME's specificity consists in proximity. Many studies have shown that SMEs prefer to maintain proxemic relations through their manager. In this article, we aim to refine our vision, trying to show the impact of proximity on perceived uncertainty by SMEs. Based on a quantitative study of 239 SMEs and statistic exploratory analyses, we will try to apprehend following research suppositions: proximity reduces uncertainty, and proximity contributes to performance. The obtained results will lead us to evaluate the importance of proximity.
\end{abstract}

\title{
RESUMEN
}

Las investigaciones cientificas referentes a las pequeñas y medianas empresas, PyMEs, sugieren una especificidad induciendo modalidades de gestion particulares de las PyMEs. Sin embargo, sobre cuales recursos se funda esta especificidad? La tesis defendida sera que la especificidad de la gestion de las PyMEs es la proximidad. Numerosos estudios han demostrado la preferencia de las PyMEs, por el intermediario de sus propietarios-directivos, para relaciones de proximidad. Trataremos de afinar esta vision procurando de ver el impacto de esta proximidad en lo que se refiere a la percepcion de incertidumbre por parte de las PyMEs. Sobre la base de un estudio quantitativo llevado a cabo acerca de 239 PyMEs y de tests estadisticos a dimension exploratorios, intentaremos de concebir dos proposiciones de investigación : la proximidad es un reductor de incertidumbre y la proximidad es un factor de performancia. Los resultados obtenidos nos llevaran a contemplar la importancia de las relaciones de proximidad.

\section{ZUSAMMENFASSUNG}

Die gängige Forschung von KMU schlägt den Kleinunternehmen spezifische Methoden der Unternehmensführung vor. Auf welchem Prinzip basieren aber diese Methoden? Die vertretene These argumentiert mit der unternehmerischen Nähe als entscheidendes Kriterium. Verschiedene Forschungen haben gezeigt, dass die Nähe der Eigentümer zu ihren Mitarbeitern ein wichtiges Merkmal in vielen KMU ist. Das Ziel dieser Arbeit ist, den Einfluss dieser unternehmerischen Nähe auf die Wahrnehmung der Unsicherheit in KMU zu erklären. Durch eine quantitative Studie von 239 KMU und darauf basierenden, explorativen statistischen Analysen, sollen zwei Forschungsvorschläge festgehalten werden: Die unternehmerische Nähe ist erstens ein Mittel um die Unsicherheit zu reduzieren und beeinflusst zweitens direkt die Leistung. Die gesammelten Resultate sollen dabei helfen, die Bedeutung der Nähe in unternehmerischen Beziehungen zu verdeutlichen. 


\section{Introduction}

«La PME n'est pas une grande entreprise miniature est certainement la phrase la plus souvent citée dans les chapitres introductifs des ouvrages et des articles consacrés à la gestion des PME. Au fil du temps, la thèse de la spécificité de gestion s'est imposée comme un véritable paradigme structurant toute la recherche en PME et légitimant la constitution de revues scientifiques et d'associations de chercheurs comme l'ICSB (International Council for Small Business) et l'ECSB (European Council for Small Business) dans le monde anglo-saxon ou l'AIREPME (Association internationale de recherche en entrepreneuriat et PME) dans le monde francophone.

Si la thèse de la spécificité est aujourd'hui très largement admise (Julien et Marchesnay, 1988; Julien, 1998; Curran et Blackburn, 2001), elle présente toutefois de multiples limites. Elle ne définit pas précisément la particularité de la PME. La thèse de la spécificité est floue et c'est vraisemblablement pour cette raison qu'elle fédère tant de chercheurs. Qui pis est, elle deviendrait dogmatique si elle inclinait à penser que toutes les entreprises de petite taille étaient de nature différente des grandes entreprises. Le courant de la dénaturation nous rappelle combien des entreprises de petite taille peuvent, dans certains contextes, fonctionner comme des grandes entreprises (Torrès, 1998; Messeghem, 2003; Torrès et Julien, 2005) et, de ce fait, relativisent la portée générale de la spécificité de gestion des PME. Enfin, la thèse de la spécificité est ambivalente car si la PME est spécifique, on doit aussi admettre que la grande entreprise est spécifique. Or, l'emploi du même terme de spécificité pour décrire deux réalités fondamentalement différentes n'est pas scientifiquement satisfaisant. Il est donc nécessaire de qualifier chacune de ces spécificités en leur donnant une définition plus précise.

La thèse défendue sera que la spécificité de gestion des PME est la proximité. Cette perspective théorique a déjà fait l'objet de travaux empiriques dans le domaine de l'éthique (Courrent et Torrès, 2005). Cette fois-ci, nous voudrions la tester dans le domaine de la perception de l'incertitude. Ainsi, les issues de cette recherche permettront d'envisager les particularités de gestion des PME en introduisant la proximité comme point focal.

Après avoir succinctement présenté la spécificité des PME à partir de la loi proxémique de Moles et Rohmer (1978), l'article exposera comment ces effets de «proxémie» se manifestent dans la perception de l'incertitude du dirigeant de PME. Ils montrent notamment que le degré de proximité affecte significativement la perception de l'incertitude selon la nature du partenaire (salarié, client, fournisseur, concurrent, État, etc.). On peut déduire de ces résultats que la perception de l'incertitude est profondément égocentrée. 


\section{La loi proxémique de Moles et Rohmer (1978)}

Moles et Rohmer (1978) proposent une conception subjective de l'espace, centrée «sur l'être individuel, unique et privilégié pour lequel "les autres" ne sont que les compléments facultatifs du Moi». Cette conception «égocentrée » de l'espace est appelée «philosophie de la centralité et correspond au point de vue "Ici et Maintenant" de l'individu en situation (espace vécu), qui éprouve son propre rapport à l'environnement. Dans cette perspective, l'être, c'est-à-dire chacun de nous, s'éprouve comme le centre du monde et celui-ci s'étend autour de lui»(Schwach, 1993). «Moi, Ici et Maintenant, je suis le centre du monde et toutes choses s'organisent par rapport à moi dans une découverte fonction de mon audace. Un monde centré sur Moi ne se peuple d'êtres et d'événements qu'à la mesure de ma perception. Ce que nous appelons une proxémique, c'est l'importance des êtres, des choses et des événements y diminuant nécessairement avec la distance à mesure que décroît leur perception elle-même» (Moles et Rohmer, 1978).

C'est ainsi que Moles et Rohmer (1978) définissent la notion de loi proxémique 1 . Selon ces derniers, «fondamentalement, axiomatiquement, ce qui est proche est, toutes choses égales d'ailleurs, plus important que ce qui est loin, qu'il s'agisse d'un événement, d'un objet, d'un phénomène ou d'un être» (figure 1a). La loi proxémique apparaît comme un principe d'ordonnancement qui hiérarchise le degré d'importance des actions et des réflexions de l'individu.

\section{Figure 1}

\section{La loi proxémique (1a) et le phénomène de paroi (1b) de Moles et Rohmer}

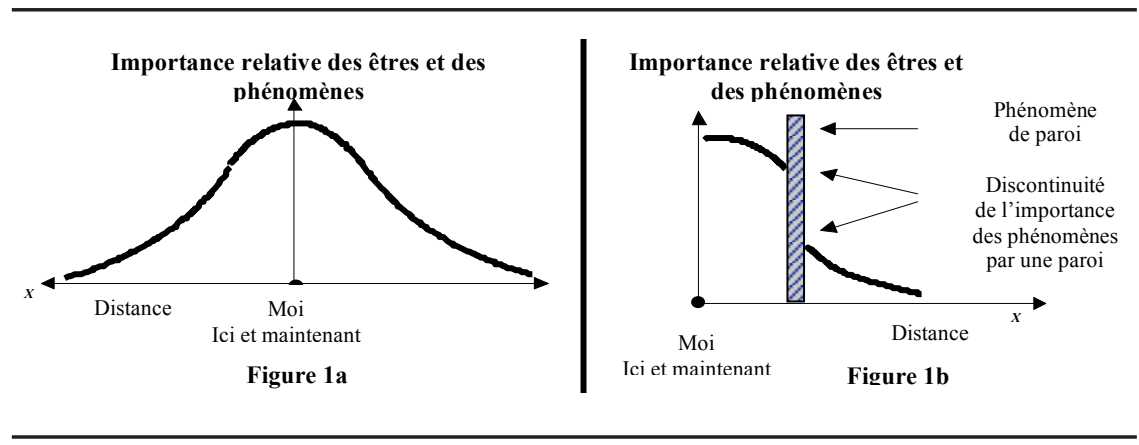

1. La proxémique est un terme inventé par Hall qui signifie l'étude de la perception et de l'usage de l'espace par l'Homme (Hall, 1981). 


\section{La pertinence de la loi proxémique dans la compréhension de la spécificité de la PME}

Pour ceux qui s'intéressent à la gestion des entreprises de faible taille (petites entreprises et toutes petites entreprises), cette axiomatique est intéressante à double titre: d'une part, elle relève de la psychologie (Moles, 1976; Schwach, 1993), ce qui constitue un champ particulièrement propice pour comprendre le comportement stratégique et organisationnel des entreprises de petite taille (Gervais, 1978; Parent, 1978) et, d'autre part, elle se fonde sur la centralité d'un point de référence unique (le Moi, Ici et Maintenant). Or, cet aspect convient parfaitement à la situation des PE et TPE, car on reconnaît généralement qu'une de leurs spécificités est l'existence du rôle prépondérant du dirigeant-propriétaire qui constitue ainsi un point de référence central par excellence (Marchesnay, 1991; d'Amboise, 1993; Julien, 1998). On retrouve ici la fameuse expression de Gumpert et Boyd (1984), «Me, myself and I». La conception égocentrée de l'espace de Moles et Rohmer paraît judicieuse pour expliquer les mécanismes de fonctionnement d'une gestion fortement personnalisée. «Dans la mesure où le groupe dirigeant est très étroit, à la limite composé d'une seule personne, l'importance des psychologies et des caractères personnels sera sans doute plus grande» dans les petites entreprises que dans les grandes (Parent, 1978, p. 92).

Plusieurs travaux relatifs à la gestion des PME révèlent l'existence de tels mécanismes hiérarchiques fondés sur la proximité:

- Dans le choix des pays d'exportation: l'attaque des marchés internationaux semble obéir à un principe de proximité comme l'a montré l'école suédoise d'Uppsala et son concept de distance psychique (Johanson et Vahlne, 1977). Le principe est simple. Lorsqu'une PME décide d'exporter, elle exporte généralement d'abord à destination des pays proches (proche sur le plan de la langue, de la culture, de la législation, des pratiques commerciales, du niveau d'éducation, etc.) puis, dans un second temps, elle se tourne vers les pays plus lointains. «Les études sur le processus d'ouverture géographique des entreprises et, en particulier des PME, soulignent le rôle de la distance psychique entre les marchés. Ainsi, les entreprises norvégiennes qui se sont internationalisées ont d'abord visé le marché anglais. Les PME danoises s'adressent essentiellement à leurs voisins de l'Union européenne et pour près de $20 \%$ à la Norvège et à la Suède; les firmes canadiennes s'orientent d'emblée vers le voisin américain. Il en est de même des entreprises turques qui concentrent leurs exportations vers un nombre limité de pays limitrophes» (Joffre, 1994, p. 13). 
- Dans le choix du successeur-repreneur: même si «dans l'esprit des dirigeants, les alternatives ne sont pas exclusives et inscrites dans un ordre immuable» (Haddadj et d'Andria, 2001), le dirigeant de PE qui désire céder son entreprise est souvent soumis à ce que nous pourrions appeler une «hiérarchisation des choix successoraux» : préférence pour la famille, puis les salariés, les clients ou les fournisseurs et, enfin, les tiers. Cette hiérarchisation se décompose en trois segments (Bah, 2002): le segment interne (les héritiers et les proches du dirigeant, les cadres ou tout ou partie du personnel de l'entreprise, etc.) puis le segment immédiat (les clients, les fournisseurs, les confrères, l'expert-comptable, etc.) et le segment externe (il correspond au marché ouvert à des tiers inconnus). Selon Bah (2002), les deux premiers segments constituent le marché «fermé», fonctionnant essentiellement sur le mode de la confidence et du «bouche à oreille » et seul le troisième segment constitue un véritable marché «ouvert». Mais ce dernier souffre d'une mauvaise image car les entreprises présentes sur ce marché sont souvent perçues comme des «canards boiteux» par les repreneurs ${ }^{2}$.

- Dans le choix de croissance: de nombreux travaux établissent une hiérarchisation, parfois de manière implicite, parfois plus nettement, des directions de la croissance de l'entreprise (Parent,1978; Churchill et Lewis, 1983; Godener, 1995). La croissance semble d'abord se réaliser au sein du même secteur, au sein de la même activité de la firme. Plus la taille est petite, plus l'entreprise a intérêt à rester confinée dans son créneau, ce que Penrose (1959) a clairement évoqué dans sa théorie des interstices. Ce n'est souvent que dans un second temps que l'on voit apparaître des choix de croissance au sein de la filière d'activités de l'entreprise, en amont et/ou en aval, ce que Parent (1978) appelle la croissance verticale. Enfin, c'est généralement en fin de parcours que la croissance se poursuit sous

2. Dans une étude réalisée par OSEO-BDPME (2005, p. 29) sur la transmission des $\mathrm{PME}$, les liens de proximité entre le repreneur et l'entreprise reprise s'avèrent fortement réducteurs ou amplificateurs de risque. Ainsi, sur une base 100 représentant l'ensemble de la population étudiée, le risque lié à une transmission familiale est estimé à 27 et celui d'une reprise par un ou des salariés à 80 . En revanche, lorsque le repreneur est sans lien avec l'entreprise, le risque s'accroît à 126 . On peut donc, sur la base de ces chiffres, affirmer que le lien de proximité est un facteur qui influence le risque de transmission. Plus la proximité entre le repreneur et le cédant est forte, plus le risque est limité. Un autre résultat intéressant concerne le degré de proximité du secteur. Lorsque le repreneur provient du même secteur que l'entreprise rachetée, le risque est réduit de $10 \%$. En revanche, lorsque le repreneur n'affiche aucune proximité sectorielle, le risque s'accroît de $50 \%$.

Revue internationale P.M.E., vol. 21, nº 1, 2008 
forme de diversification, soit en termes de métier, soit en termes de mission. La diversification totale ne devenant qu'une solution de dernière extrémité car elle est la plus risquée. On retrouve dans ces étapes de la croissance un ordre implicite qui commence par la croissance horizontale («le mode le plus normal, le plus fréquent et le plus habituel de la croissance des firmes» selon Parent, 1978), puis se poursuit par la croissance verticale (la filière) et enfin, se complète par la croissance conglomérale. Bien que cet ordre ne soit pas toujours systématique et que des facteurs comme la technologie puissent en modifier l'intensité (Godener, 1994), force est de constater que les choix en matière de direction de la croissance attestent d'une loi proxémique signifiant que la croissance de l'entreprise se met en œuvre du plus proche (le créneau, l'interstice) vers le plus lointain, le plus risqué (la diversification totale). On peut faire le même constat d'une loi proxémique en ce qui concerne les modalités de la croissance (croissance interne versus croissance externe). Plus la taille est petite, plus le dirigeant a tendance à privilégier la croissance interne à la croissance externe (Parent, 1978).

- Dans les choix de recrutement: de manière générale, plus l'entreprise est de petite taille, plus elle a tendance à privilégier les liens de proximité dans sa gestion du personnel. Dans une enquête concernant la gestion du personnel dans les petites entreprises, Chassard et Bouruet (2003) montrent que les relations et le «bouche à oreille» arrivent en tête des réponses, bien devant les agences d'intérim et les cabinets de recrutement. Le dirigeant de la petite entreprise n'aime pas les intermédiaires et préfère mobiliser son propre carnet d'adresses pour recruter quelqu'un. Ce type de comportement est tout à fait rationnel car une recommandation par un proche est un gage de confiance. La proximité joue donc un rôle de garantie mais aussi de réducteur d'incertitude. C'est la raison pour laquelle lorsque le dirigeant de PME embauche, il privilégie souvent son conjoint ou sa conjointe, puis élargit sa recherche à la famille ou au cercle des amis. Ce n'est qu'en dernier ressort qu'il recourt au marché du travail, plus anonyme. La famille, les amis et l'entourage constituent donc des ressources stratégiques lorsque le dirigeant déclare les utiliser comme une source principale de travail. C'est l'importance de ces relations humaines de proximité qui fait dire très justement à Letowski (2003) que le concept de ressources humaines n'a pas de sens dans les très petites entreprises sauf à tenir compte des ressources « hors salariat» que sont le conjoint, les enfants, les associés, les pairs, etc. Ce comportement proxémique est également mis en évidence par les travaux 
de Moreau (2006) en ce qui concerne le recrutement d'un associé. Ce dernier s'est intéressé à la formation des équipes d'entrepreneurs. Bien que distinguant deux catégories de raisons qui guident la constitution d'une équipe (l'une de type stratégique fondée sur le calcul économique et la complémentarité, l'autre de type social fondée sur la proximité et la similarité), l'étude empirique, portant sur 30 équipes, montre une nette prédilection pour le principe de proximité. Selon Moreau, «les personnes cherchent d'abord la confiance, qui est justement procurée par l'existence des liens antérieurs d'ordre familiaux ou amicaux. Voilà pourquoi le choix d'un proche plutôt que d'un inconnu est une option intéressante, voire privilégiée, même si l'inconnu possède un excellent profil professionnel » (Moreau, 2006, p. 64). Ainsi, l'auteur conclut en considérant que «la proximité sociale structure le choix du cofondateur plus fortement et plus fréquemment que les injonctions directes issues d'une volonté stratégique d'optimisation économique» (Moreau, 2006, p. 65).

- Dans le choix des modes de financement: le premier problème financier auquel est confronté un dirigeant de PME est la constitution de son capital d'origine. À cet égard, on peut constater que la quasitotalité des travaux considère que ce capital est fortement soumis aux effets de proximité. Par exemple, Crevoisier (1997) introduit le concept de «capital de proximité» qui correspond aux capitaux provenant principalement des fonds personnels du dirigeantpropriétaire apportés au moment de la création de l'entreprise et des fonds qu'il aura pu emprunter aux membres de sa famille et à ses proches. St-Pierre $(1999$, p. 212) évoque à son tour le concept de «capital amical» qu'elle définit comme le capital qui «provient des parents ou amis proches de l'entrepreneur, ou de toute autre personne ayant des relations de proximité avec lui. Ce capital est souvent utilisé au démarrage de l'entreprise et les exigences sont relativement souples». Lorsque le dirigeant de PME envisage de recourir à de l'emprunt bancaire, on retrouve encore ces effets de proximité. «Les résultats de certaines enquêtes montrent que les dirigeants ont tendance à ne faire affaire qu'avec quelques institutions financières (souvent une seule). Il est plus rentable de développer de bonnes relations avec un banquier, lequel finit par comprendre la personnalité du propriétaire-dirigeant et accepte son processus décisionnel particulier» (St-Pierre et Beaudoin, 1995). Binks et Ennew (1997) vont encore plus loin en préconisant une «relation participative» entre les PME et leur banque : l'information parfaite est un objectif inaccessible mais de proches relations de travail entre la banque et l'entreprise peuvent significativement améliorer 
l'information disponible et réduire les effets négatifs de l'asymétrie d'informations (Binks et Ennew, 1997, p. 90). Le concept de «territoire financier » forgé par Belletante (1991) va dans le même sens et atteste de la primauté de la proximité.

Pour résumer, les choix des modes de financement en PME révèlent que la croissance est financée prioritairement par autofinancement puis par dettes bancaires plutôt que par émissions de capital-actions (ouverture du capital) [Norton, 1991; St-Pierre et Beaudoin, 1995; Mahérault, 1999]. Cette séquence des modes de financement suggère une hiérarchie fondée sur la proximité que l'on pourrait appeler une «proxemic order theory».

- Dans le choix du réseau d'appui et de soutien: la théorie des couches («layers theory») de Gibb (1988) témoigne à nouveau de la hiérarchisation proxémique entre les différentes sphères composant le réseau de support du dirigeant de PME (figure 2). Selon Gibb (1988), «a small business will be subject to many influences. It is not however always obvious what these influences will be or what will be the interplay between the different influences ». Ainsi, Bridge, O'Neill et Cromie (1998) pensent, en reprenant Gibb, que les couches les plus proches auront le plus d'influence. Gibb considère que l'influence du réseau d'appui est hiérarchisée en fonction de la proximité et en tire les conséquences suivantes: «If friends when consulted are negative in their advice then it will be far more influential than any positive input from government agencies. » Les avis et les influences ne sont pas égaux mais hiérarchisés selon une pondération qui s'accroît en fonction de la proximité du lien avec le propriétaire-dirigeant.

Figure 2

La théorie des couches

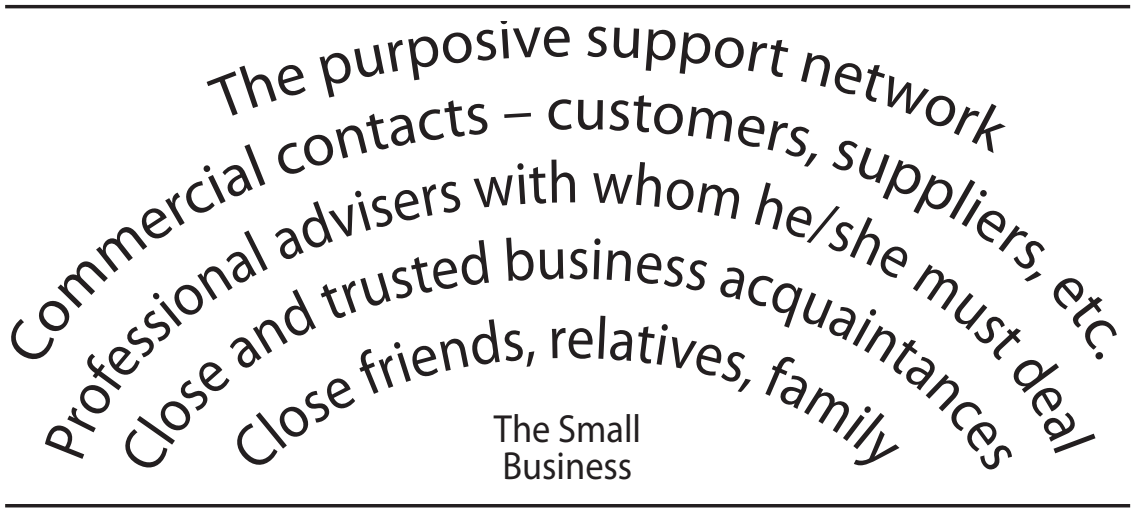

Source: Gibb, 1988, dans Bridge, O’Neill et Cromie, 1998.

Revue internationale P.M.E., vol. 21, nº 1, 2008 
Ces six exemples présentent plusieurs points communs. D'une part, ils illustrent tous l'existence d'une hiérarchie graduée du plus proche au plus lointain ${ }^{3}$. Cette graduation correspond à un phénomène proxémique. D'autre part, ces exemples concernent tous une décision dans un domaine précis (décisions d'exporter, de transmettre, de se développer, de recruter, de financer et de choisir un soutien). Ces travaux semblent tous converger vers l'idée générale que le dirigeant de PME est soumis implicitement à un principe de proximité dans son mode de décision, quelles que soient les circonstances. En ce sens, si l'on généralise le propos, on peut en déduire que la petitesse des entreprises induit un mode de décision proxémique.

Mais qu'en est-il des phases précédentes à l'acte de décision? On sait depuis Herbert Simon (1980) que la décision n'est que l'étape ultime d'un processus beaucoup plus large qui se décompose en trois phases IDC (Intelligence - Design - Choice). Selon Simon (1947), comme l'optimisation est trop compliquée, l'homme dont la rationalité est limitée se contente généralement de solutions satisfaisantes.

Si le propos initial de Simon a pour vocation de concerner les décideurs des structures administratives, on peut déceler certains passages qui renforcent la thèse d'une rationalité plus fortement limitée en PME: «le comportement d'un individu unique, isolé, ne peut en aucun cas atteindre un niveau de rationalité élevé» (Simon, 1947, p. 71). Comme la PME se caractérise par un décideur unique, on peut en déduire que la rationalité en sera d'autant plus limitée. Or, pour compenser ce handicap de taille et à la lueur des exemples précédents, nous constatons que les solutions satisfaisantes sont très souvent en PME les solutions les plus proches. La proximité semble même correspondre à ce que Simon (1947, p. 86) appelle un «mécanisme de continuité »: «Une fois orientés dans une direction particulière, l'attention et le comportement ont tendance à persister durablement dans la même voie.» Dans de nombreux domaines, la proximité semble orienter, voire canaliser le comportement du dirigeant de PME. À tel point que le fameux «satisfacing behavior» de Simon peut être reformulé plus précisément dans le cas du dirigeant d'une PME comme un «proxemicing behavior» (contraction des termes «proxemic» et «satisfacing»). Ainsi, la proximité pourrait être une explication fondamentale du principe de rationalité limitée ${ }^{4}$.

3. On observera plusieurs contre-exemples à cette conception proxémique. Les travaux sur les INV (International New Venture), sur les entreprises à croissance rapide ou start-ups, sur le capital-risque, etc., montrent que certaines entreprises de petite taille échappent totalement à cette conception. Mais ces PME demeurent encore des exceptions.

4. Selon Demailly (2004, p. 28-29), à «l'homme économique d du courant néoclassique, qui maximise rationnellement ses utilités, Herbert Simon oppose un «homme

Revue internationale P.M.E., vol. 21, nº 1, 2008 
Dans ces conditions, on peut imaginer que si les décisions suivent un ordre implicite dicté par des considérations de proximité, les perceptions doivent subir la même influence et donc, le même cheminement. En effet, si l'on observe dans des domaines aussi divers que l'exportation, le financement, la croissance, etc., que les décisions suivent une séquence proxémique, on peut étendre la portée de ce principe et faire l'hypothèse qu'en amont de la décision proprement dite, la collecte d'information et la perception des dirigeants de PME sont elles aussi sensibles aux effets de proximité. En d'autres termes, on peut avancer l'idée que la proximité joue sur tout le processus de décision, depuis la phase d'intelligence d'un problème jusqu'au choix définitif en passant par la phase de conception.

Ainsi apparaît une proposition de recherche qui a rarement été testée dans le champ des PME: analyser comment les perceptions des dirigeants de PME sont influencées par les effets de proximité. La suite de ce travail a pour objet d'explorer le rôle et l'importance de cette hiérarchie proxémique dans la perception de l'incertitude de la part des dirigeants de PME.

\section{3. Étude de la relation proximité - incertitude}

De manière générale, l'incertitude correspond au manque d'information concernant les facteurs environnementaux, rendant impossible la prévision de l'impact d'une décision spécifique sur l'organisation, et à l'impossibilité de donner des probabilités d'événements quant à l'impact des facteurs environnementaux sur l'organisation (Duncan, 1972). De la même façon, Demsetz (1998) estime que l'incertitude sera présente lorsque l'information est suffisamment défaillante pour qu'il soit impossible de faire des estimations de probabilités des différents événements possibles. D'ailleurs, dans certains cas extrêmes, l'incertitude considérée comme radicale va s'identifier à l'ignorance (Moureau et Rivaud-Danset, 2004, p. 10).

De ces quelques définitions générales, nous pouvons faire l'hypothèse que la proximité est un facteur de réduction d'incertitude. Plusieurs raisons permettent de défendre cette hypothèse centrale.

administratif» (limité par son environnement externe et ses propres capacités mentales) qui se contente de prendre des décisions «satisfaisantes» en se basant sur des repères plus psychologiques ou sociaux que rationnels ou logiques. L'un de ces repères est l'identification aux sous-objectifs de l'entourage immédiat (collègues ou clients): «plutôt que de viser les objectifs généraux de l'organisation, on s'assure que la décision qui sera prise est acceptable par l'entourage». La notion d'entourage renvoie bien à un environnement de proximité. On peut ici faire l'hypothèse que la proximité est un construit stratégique et organisationnel rationnel qui permet au dirigeant d'une PME de maintenir son emprise sur l'entreprise et son évolution.

Revue internationale P.M.E., vol. 21, nº 1, 2008 
Si l'on définit l'incertitude comme l'impossibilité de donner a priori une probabilité de survenance à un phénomène, on acceptera l'idée selon laquelle plus l'horizon temporel s'élargit, plus l'incertitude croît. «C'est ainsi que le temps qu'il fera dans un avenir proche n'est selon Keynes que modérément incertain [...] Suivant cette conception, c'est le futur et, en particulier, le long terme qui sont incertains » (Moureau et Riveau-Danset, 2004, p. 10). Si l'on sait avec une certitude raisonnable ce que l'on va faire dans une heure ou deux, il devient quasiment impossible de probabiliser ce que l'on fera dans deux ans, a fortiori dans vingt ans. Si l'on mesure la proximité sur une échelle de temps, on peut admettre que plus la proximité temporelle est forte, plus l'incertitude diminue.

C'est une des raisons principales qui incitent le dirigeant d'une PE à raisonner très souvent à court terme, privilégiant les solutions rapidement efficaces au détriment de solutions d'ordre stratégique dont les effets sont moins immédiats. Ce phénomène correspond à ce que Mahé de Boislandelle (1996) appelle «l'effet de microcosme». C'est ainsi que le champ d'action d'un dirigeant de PME se limite souvent à l'environnement le plus proche, car c'est à ce niveau que le dirigeant pense avoir la plus grande marge de manœuvre et disposer d'une plus grande contrôlabilité des conséquences de ses actions. Cela tient à deux raisons:

- d'une part, la proximité est un élément qui renforce les liens interpersonnels et qui, de ce fait, améliore la circulation de l'information et donc réduit l'incertitude. Seule la proximité permet de déchiffrer l'information tacite ou implicite. «De près, on se comprend mieux», dit le vieil adage;

- d'autre part, la proximité est souvent un réflexe en cas de situation de crise. Devant un péril ou une situation imprévue, la réaction spontanée consiste à se « raccrocher aux branches » et à s'en tenir à ce que l'on sait faire et à ce que l'on connaît le mieux.

Ainsi, on voit bien apparaître le lien entre proximité et incertitude. Plus le dirigeant se focalise sur les éléments proches de lui, plus il renforce son contrôle et plus il réduit l'incertitude. Le principe de proximité est, selon nous, à la fois une explication du principe de rationalité limitée et une réponse adaptée à l'asymétrie d'information. Ce type de comportement privilégiant la proximité est tout à fait rationnel car une recommandation par un proche ou une relation d'affaires avec une connaissance est un gage de confiance. La proximité joue ici un rôle de garantie mais aussi de réducteur d'incertitude. Elle réduit l'asymétrie d'information et élargit la rationalité. L'un des enseignements que l'on peut déduire des travaux d'Akerlof (1970) est que le vendeur d'une voiture d'occasion en bon état a davantage intérêt 
à revendre son véhicule à quelqu'un qu'il connaît qu'à un inconnu car la proximité, par la confiance qu'elle peut engendrer, atténue le problème de l'asymétrie d'information.

D'où nos propositions de recherche. La première vise à vérifier si l'incertitude est soumise à un effet de proximité. Nous testerons cet effet de proximité en deux temps. Tout d'abord, nous vérifierons si le degré de proximité réduit l'incertitude. Puis, conformément aux travaux de Torrès (2003), selon lequel plus la taille se réduit, plus les effets de proximité se renforcent, nous testerons l'effet-taille. En d'autres termes, nous verrons si les effets de proximité s'amplifient lorsque la taille se réduit. Enfin, conformément à l'esprit des sciences de gestion, sciences de la performance, nous chercherons à vérifier les liens éventuels entre la proximité, l'incertitude et la performance.

C'est ainsi que notre première proposition de recherche sera:

\section{Proposition 1: la proximité est un réducteur d'incertitude.}

Et nous opérationnaliserons cette proposition par deux hypothèses que nous testerons. Ces hypothèses sont:

H1a: plus l'acteur est proche, moins il est considéré comme incertain. En effet, nous pouvons supposer que la proximité introduit une meilleure connaissance de l'acteur, ce qui induit une réduction de l'incertitude.

H1b: plus l'entreprise est petite, plus sa perception de l'incertitude des acteurs environnementaux sera forte. Comme le suggèrent les concepts de microcosme de Mahé de Boislandelle (1996) et de «proxémie aiguë» de Torrès (2003), on peut supposer que la petitesse des entreprises induit une perception incertaine de l'environnement et une préférence pour le proche.

Notre seconde proposition de recherche, qui intègre la dimension de performance, sera:

\section{Proposition 2: la proximité est un facteur de performance.}

De façon similaire, deux hypothèses nous permettront l'opérationnalisation de cette seconde proposition:

$\mathrm{H} 2 \mathrm{a}$ : plus les acteurs proches sont considérés comme certains, meilleure sera la performance. Nous supposerons qu'une limitation de l'incertitude de la part des acteurs les plus proches est source d'avantages 
pour les entreprises, notamment dans le cadre des PME, car la proximité semble être un facteur qui améliore la confiance, la solidarité, l'écoute, la fiabilité, etc.

$\mathrm{H} 2 \mathrm{~b}$ : plus l'entreprise est petite, plus le lien entre mauvaise performance et incertitude des acteurs proches sera fort. Cette hypothèse vise à tester $\mathrm{H} 2 \mathrm{a}$ en fonction d'un effet-taille car les spécificités des plus petites entreprises amplifient le rôle des acteurs proches.

\section{Méthodologie et résultats}

L'échantillon d'étude est constitué de 239 PME françaises issues de plusieurs secteurs d'activité. L'effectif moyen est de 18 employés et l'âge moyen est évalué à six années. Nous noterons que cet échantillon comporte $68 \%$ de TPE (163 entreprises inférieures à 10 employés et 76 entreprises ayant un effectif supérieur ou égal à 10 employés). L'administration du questionnaire s'est effectuée par Internet auprès de dirigeants de PME. Nous allons nous livrer à différents tests statistiques classiques (de corrélations, de comparaison de moyennes) afin de vérifier nos quatre hypothèses de recherche et, par-delà, nos deux propositions concernant le rôle de la proximité au sein des entreprises de petite dimension. Cependant, notre dispositif de recherche, conditionné par les variables mesurées, la nature de nos interrogations, la taille de l'échantillon et les outils utilisés, aura une vocation essentiellement exploratoire. Nous chercherons à relever des liens éventuels afin de décrire les relations entre proximité et incertitude dans le cadre des PME sans chercher à valider des relations confirmatoires au sein d'une modélisation exhaustive. Notre travail se veut une première étape dans un processus de recherche plus complet.

\subsection{Proximité et incertitude}

Nous cherchons à vérifier :

H1a: Plus l'acteur est proche, moins il est considéré comme incertain.

Il était demandé d'indiquer sur une échelle de cinq points (de très incertain à très certain) quel était le niveau d'incertitude associé à chacun des acteurs suivants: État, concurrents, clients, fournisseurs, employés. Les résultats obtenus sont les suivants (tableau 1), dans l'ordre descendant du niveau d'incertitude perçu. 
TABleau 1

Importance de la perception de l'incertitude par acteur

\begin{tabular}{lccccc}
\hline & N & Minimum & Maximum & Moyenne & Écart type \\
\hline Incertitude des employés & 239 & 1,00 & 5,00 & 2,3598 & 0,87 \\
Incertitude des fournisseurs & 239 & 1,00 & 5,00 & 2,6192 & 0,87 \\
Incertitude des clients & 239 & 1,00 & 5,00 & 2,9247 & 0,95 \\
Incertitude des concurrents & 239 & 1,00 & 5,00 & 2,9582 & 1,01 \\
Incertitude de l'État & 239 & 1,00 & 5,00 & 3,3347 & 1,16 \\
\hline
\end{tabular}

Nous pouvons donc constater un écart entre un acteur éloigné (l'État) et un acteur proche (les employés). Les employés, plus proches, sont perçus comme moins incertains que l'État, plus éloigné. À ce titre, un test de comparaison de moyennes pour échantillons appariés (test $t$ ) nous permet de constater que cette différence est significative (à 0,01$)$. Dès lors et en regard des moyennes obtenues, nous pouvons penser qu'un regroupement des différentes perceptions concernant l'incertitude des acteurs est possible.

Notre méthodologie reposera sur le caractère multidimensionnel de nos mesures. En d'autres termes, sommes-nous en présence d'une échelle unique mesurant l'incertitude des acteurs ou pouvons-nous identifier des sous-échelles, en termes de distance de l'acteur?

TABLEAU 2

Corrélations entre les perceptions d'incertitude par acteurs

\begin{tabular}{lcllll}
\hline & $\begin{array}{l}\text { Inc. des } \\
\text { employés }\end{array}$ & $\begin{array}{l}\text { Inc. des } \\
\text { fournisseurs }\end{array}$ & $\begin{array}{l}\text { Inc. des } \\
\text { clients }\end{array}$ & $\begin{array}{l}\text { Inc. des } \\
\text { concurrents }\end{array}$ & $\begin{array}{l}\text { Inc. de } \\
\text { l'État }\end{array}$ \\
\hline Incertitude des employés & 1 & & & & \\
Incertitude des fournisseurs & 0,121 & 1 & & & \\
Incertitude des clients & 0,125 & $0,270^{* *}$ & 1 & & \\
Incertitude des concurrents & 0,085 & $0,130^{*}$ & $0,146^{*}$ & 1 & \\
Incertitude de l'État & 0,034 & 0,127 & 0,008 & 0,087 & 1 \\
\hline
\end{tabular}

* La corrélation est significative au niveau 0,05 .

** La corrélation est significative au niveau 0,01 .

Cette analyse des corrélations inter-items (tableau 2) est intéressante puisqu'elle permet de remarquer un lien significatif entre l'incertitude des concurrents, des clients et des fournisseurs tandis que l'incertitude de l'État et celle des employés ne sont pas à relier aux autres incertitudes perçues. À ce titre, une analyse en composante principale (ACP) nous permet également de constater que l'incertitude de l'État correspond à une dimension isolée avec une contribution spécifique élevée $(0,88)$. Ainsi, nous pouvons considérer que l'incertitude de l'État est celle qui est la plus distante des autres perceptions d'incertitude. 
Du fait des deux résultats issus de l'analyse de corrélations et de l'analyse en composante principale et afin de simplifier notre analyse, nous allons modifier notre mesure de l'incertitude des acteurs au regard de trois items:

- Incertitude des acteurs éloignés (État).

- Incertitude des acteurs intermédiaires (clients, concurrents, fournisseurs) $)^{5}$.

- Incertitude des acteurs proches (employés).

Nous obtenons donc une classification de l'importance de l'incertitude perçue qui prend la forme suivante (figure 3).

Figure 3

Classification de l'incertitude perçue par acteur

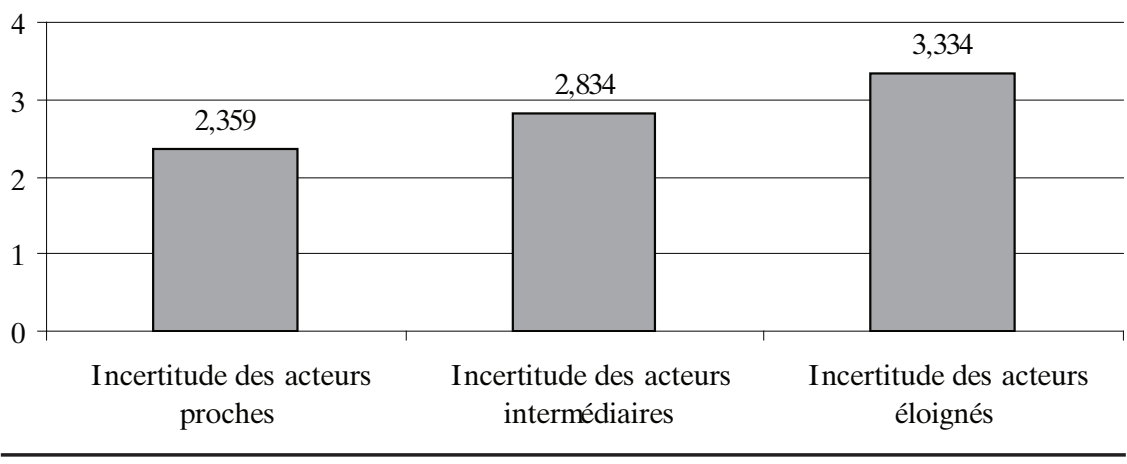

Notons qu'un test de comparaison de moyennes (test $t$ ) révèle une différence significative entre ces trois scores (seuil de significativité à 0,01 pour les tests de différence de chacune des combinaisons possibles). Cette représentation graphique nous permet d'envisager clairement la relation positive entre l'éloignement de l'acteur et son incertitude perçue associée. En d'autres termes, nous pouvons envisager l'idée que plus l'acteur est éloigné, plus il est considéré comme incertain et qu'inversement la proximité semble réduire l'incertitude.

Ainsi, l'hypothèse H1a est validée. En contexte PME, plus l'acteur est éloigné, plus il est considéré comme incertain. Ce résultat est à rapprocher de ceux de Courrent et Torrès (2005) qui ont observé une hiérarchie quasi

5. Comme la mesure de l'incertitude des acteurs intermédiaires comporte peu d'items, nous n'utiliserons pas l'alpha de Cronbach afin de mesurer la consistance interne de l'échelle. 
similaire en termes de proximité et de comportement éthique (les salariés puis les clients et les fournisseurs et, enfin, l'État). Dans cette étude empirique, les deux auteurs observent également le comportement très défiant des dirigeants de PME à l'égard de l'État comme si ce dernier était une entité à part. Est-ce un trait propre au petit patronat français, lequel dans son passé a parfois «flirté » avec des mouvements corporatistes (UDCA de Pierre Poujade en 1956), n'hésitant pas à mobiliser les petits patrons dans des actions violentes, souvent contre l'État? Cependant, le haut niveau d'incertitude associé à l'acteur le plus éloigné (l'État) peut également s'expliquer, a priori, par le manque de stabilité inhérent à cet acteur (changement de politique, modification des textes de loi, évolution des priorités économiques, alternance, non-respect des engagements électoraux, etc.).

On peut également avoir une lecture juridique de cette sensibilité de l'incertitude à la proximité. Les salariés renvoient au droit du travail tandis que les concurrents, les clients et les fournisseurs s'inscrivent dans un droit des affaires et, enfin, l'État relève du droit public administratif. Nos résultats laissent entrevoir l'idée d'une hiérarchisation des différents types de droit pour un dirigeant de PME: le droit du travail (employés), le droit des affaires (clients, concurrents, fournisseurs) et le droit administratif (État).

Toujours est-il que cette répartition de l'incertitude perçue au regard de la proximité des acteurs nous permet de constater que l'incertitude des acteurs intermédiaires est corrélée positivement et significativement avec celle des acteurs proches tandis que l'incertitude des acteurs éloignés n'est liée à aucune autre incertitude provenant d'autres acteurs, plus proches. Le tableau de corrélation entre l'incertitude des différents acteurs nous permettra de quantifier cette perspective (tableau 3).

\section{TABLEAU 3}

Corrélations entre les perceptions de l'incertitude des différents types d'acteurs

\begin{tabular}{lccc}
\hline & $\begin{array}{l}\text { Incertitude des } \\
\text { acteurs proches }\end{array}$ & $\begin{array}{l}\text { Incertitude des } \\
\text { acteurs intermédiaires }\end{array}$ & $\begin{array}{l}\text { Incertitude des } \\
\text { acteurs éloignés }\end{array}$ \\
\hline $\begin{array}{l}\text { Incertitude } \\
\text { des acteurs proches }\end{array}$ & 1 & & \\
$\begin{array}{l}\text { Incertitude des } \\
\text { acteurs intermédiaires }\end{array}$ & $0,162^{*}$ & 1 & 1 \\
$\begin{array}{l}\text { Incertitude } \\
\text { des acteurs éloignés }\end{array}$ & 0,034 & 0,108 & 1 \\
\hline
\end{tabular}

* La corrélation est significative au niveau 0,05 . 


\subsection{L'effet-taille sur la relation proximité-incertitude}

Nous cherchons à vérifier :

H1b: plus l'entreprise est petite, plus sa perception de l'incertitude des acteurs environnementaux sera forte.

Est-ce qu'un effet-taille influence la relation incertitude-proximité des acteurs? Le test de cette hypothèse donne un résultat intéressant puisque nous ne trouvons pas de modération de l'incertitude afférente aux acteurs en fonction de la taille de l'entreprise. Le test de corrélation suivant (tableau 4) permet de constater l'absence de significativité dans les relations envisagées.

\section{TABLEAU 4}

Corrélations entre incertitude des acteurs et taille de l'entreprise

\begin{tabular}{lcccc}
\hline & $\begin{array}{c}\text { Incertitude des } \\
\text { acteurs proches }\end{array}$ & $\begin{array}{c}\text { Incertitude des } \\
\text { acteurs intermédiaires }\end{array}$ & $\begin{array}{c}\text { Incertitude des } \\
\text { acteurs éloignés }\end{array}$ & $\begin{array}{c}\text { Nombre } \\
\text { d'employés }\end{array}$ \\
\hline $\begin{array}{l}\text { Nombre } \\
\text { d'employés }\end{array}$ & 0,119 & 0,077 & $-0,010$ & 1 \\
\hline
\end{tabular}

En d'autres termes, il n'est pas possible, au vu de ces résultats, de considérer que la perception d'incertitude en fonction du degré de proximité des acteurs environnementaux est variable selon la taille des PME. Il existe donc un effet absolu. Cependant, l'importance théorique de ce résultat est à modérer du fait de la spécificité de notre échantillon et de notre méthode de mesure ${ }^{6}$. Pour être plus en phase avec ces perspectives et nous assurer de cette absence de modération, nous pouvons envisager un test de comparaison de moyennes en fonction d'une catégorisation TPE / non TPE (tableau 5).

\section{TABLEAU 5}

\section{Comparaison de l'incertitude selon la taille (test $t$ )}

\begin{tabular}{|c|c|c|c|c|c|c|c|c|c|}
\hline & \multicolumn{2}{|c|}{$\begin{array}{c}\text { TPE } \\
(n=163)\end{array}$} & \multicolumn{2}{|c|}{$\begin{array}{l}\text { Non TPE } \\
(n=76)\end{array}$} & \multicolumn{2}{|c|}{$\begin{array}{c}\text { Total } \\
(n=239)\end{array}$} & \multicolumn{3}{|c|}{ Test de Student } \\
\hline & Moy. & Var. & Moy. & Var. & Moy. & Var. & DDL & $t$ & Sig \\
\hline $\begin{array}{l}\text { Incertitude des } \\
\text { acteurs proches }\end{array}$ & 2,36 & 0,90 & 2,36 & 0,80 & 2,36 & 0,86 & 237 & 0,06 & - \\
\hline $\begin{array}{l}\text { Incertitude des } \\
\text { acteurs intermédiaires }\end{array}$ & 2,79 & 0,64 & 2,93 & 0,63 & 2,83 & 0,64 & 237 & $-1,67$ & 0,1 \\
\hline $\begin{array}{l}\text { Incertitude des } \\
\text { acteurs éloignés }\end{array}$ & 3,33 & 1,15 & 3,34 & 1,20 & 3,33 & 1,16 & 237 & $-0,07$ & - \\
\hline
\end{tabular}

6. L'effet-taille mesuré est dans notre échantillon de faible amplitude puisque la plus grande entreprise compte seulement 350 salariés. Il aurait été intéressant d'avoir un plus grand nombre d'entreprises de plus grande taille. 
Nous pouvons constater, du fait de la catégorisation opérée, une significativité relativement faible, et ce, uniquement en ce qui concerne l'incertitude des acteurs intermédiaires. En d'autres termes, les TPE perçoivent de façon moins incertaine les acteurs intermédiaires de leur environnement (concurrents, clients, fournisseurs) que les PME de plus grande taille. Mais le seuil de significativité obtenu $(0,10)$ est relativement faible et ne permet pas de conclure de façon franche.

\subsection{Incertitude de l'acteur proche et performance}

La première hypothèse concernant notre seconde proposition de recherche est:

$\mathrm{H} 2 \mathrm{a}$ : plus les acteurs proches sont considérés comme certains, meilleure sera la performance.

Nous pouvons supposer que l'incertitude concernant certains acteurs est particulièrement dommageable quant à l'obtention de la performance. Afin de mesurer la nature de cette relation, nous avons établi une échelle multidimensionnelle sur la base de la perception du dirigeant. En effet, la performance peut se mesurer de façon objective ou subjective (Dess et Robinson, 1984). Chandler et Hanks (1993) constatent la difficulté d'utiliser des données objectives pour l'étude des jeunes entreprises, mais ces difficultés se posent également pour les petites entreprises. Ils remarquent que les mesures concernant une perception de la performance en termes d'augmentation ou de diminution de certains critères de résultats apparaissent comme satisfaisantes. Ces mesures peuvent être envisagées comme une alternative au recensement chiffré et précis de la performance de l'entreprise. Ainsi, la mesure de la performance se fera en fonction de six indicateurs généralement retenus (Messeghem, 1999) [rentabilité, effectif, chiffre d'affaires, qualité, motivation et réputation 7 pour lesquels, sur la base d'une échelle de cinq points, le dirigeant devra exprimer l'évolution au cours des dernières années. La mesure de la relation entre l'incertitude des acteurs selon leur nature et le niveau de performance obtenu est la suivante.

7. L'échelle initiale incluait une variable mesurant l'évolution de l'invulnérabilité de l'entreprise. Toutefois, une mesure des corrélations inter-items laissait apparaître que cette variable ne contribuait pas à la même dimension issue des autres variables. À la suite d'une mesure des corrélations inter-items effectuée sur les variables restantes, il est apparu que toutes les variables étaient corrélées significativement et positivement entre elles, la mesure de la consistance interne effectuée avec l'alpha de Cronbach donnant un résultat de 0,73. 


\section{TABLEAU 6}

Corrélations entre incertitude des acteurs et niveau de performance

\begin{tabular}{lc}
\hline & Performance \\
\hline Incertitude des acteurs proches & $-0,179 *$ \\
Incertitude des acteurs intermédiaires & 0,016 \\
Incertitude des acteurs éloignés & 0,067 \\
Performance & 1 \\
\hline
\end{tabular}

* La corrélation est significative au niveau 0,01 .

Si les acteurs intermédiaires ou éloignés ne semblent pas être en relation significative avec le niveau de performance, en revanche, plus l'incertitude des acteurs proches est forte, moins bonne sera le niveau de performance. Ainsi, nous nous rendons compte que l'incertitude associée à des acteurs environnementaux de proximité est particulièrement pénalisante quant à l'obtention de la performance. Par-delà, nous pouvons envisager l'idée selon laquelle plus l'entreprise est certaine de son environnement proche, meilleure sera sa performance. Il en ressort logiquement une importance manifeste du management de l'incertitude des acteurs proches des PME car les acteurs proches sont une ressource cruciale dans le fonctionnement de l'entreprise, plus que tout autre acteur.

\subsection{Incidence de la taille sur la relation incertitude, proximité, performance}

La dernière hypothèse de notre recherche est:

$\mathrm{H} 2 \mathrm{~b}$ : plus l'entreprise est petite, plus le lien entre mauvaise performance et incertitude des acteurs proches sera élevé.

Le tableau 7 compare les corrélations entre incertitude des acteurs et performance en fonction de l'appartenance ou non des entreprises à la catégorie des TPE. Ce résultat est d'autant plus intéressant qu'il semble exister une différence forte entre TPE et non-TPE.

Nous nous rendons ainsi compte que c'est uniquement pour les plus petites des entreprises de notre échantillon qu'un lien significatif et négatif entre incertitude des acteurs proches et niveau de performance est à constater de façon significative. Ainsi, nous pouvons penser que plus l'entreprise est de faible dimension, plus une forte incertitude des acteurs proches est à relier à une mauvaise performance. Cependant, un test de comparaison des coefficients de corrélation à partir de la transformation de Fisher (test $Z$ ) ne nous permet pas de conclure à une significativité au seuil de 0,05. 
TABLEAU 7

\section{Corrélations entre incertitude des acteurs} et niveau de performance selon la taille

\begin{tabular}{l|l|c}
\hline & & Performance \\
\hline \multirow{3}{*}{ TPE } & Incertitude des acteurs proches & $-0,215^{*}$ \\
& Incertitude des acteurs intermédiaires & 0,033 \\
& Incertitude des acteurs éloignés & 0,093 \\
\hline \multirow{3}{*}{ Non TPE } & Incertitude des acteurs proches & $-0,096$ \\
& Incertitude des acteurs intermédiaires & $-0,026$ \\
& Incertitude des acteurs éloignés & 0,014 \\
\hline
\end{tabular}

* La corrélation est significative au niveau 0,01 .

\section{Conclusion}

$\mathrm{Au}$ terme de cette étude empirique, il semble que la proximité exerce un effet de réduction d'incertitude. Cela renvoie à des résultats déjà connus qui semblent attester que la proximité est un facteur rassurant, un peu comme si «de près on se comprenait mieux ${ }^{8}$ ». Les éléments de compréhension de cet état peuvent se retrouver dans l'idée que l'acteur proche est mieux connu par le dirigeant que ne l'est l'acteur éloigné. Et comme l'incertitude s'envisage sous l'angle du manque d'information (Duncan, 1972), une meilleure connaissance de l'acteur entraînera logiquement une réduction de l'incertitude perçue afférente. Encore faut-il pouvoir articuler cette réduction de l'incertitude avec les modes de gestion des PME. Nos propositions en termes de proximité vont en ce sens. Dans ces conditions et en prolongeant nos résultats, nous pouvons nous poser de nombreuses questions. Pourquoi le proche paraît-il plus certain que l'éloigné ? Serait-il plus contrôlable?

Par ailleurs, nos résultats montrent qu'il existe un effet absolu de cette incertitude, quelle que soit la taille de l'entreprise étudiée. Les plus grandes des PME comme les plus petites d'entre elles connaissent cette hiérarchisation de l'incertitude qui croît en fonction de l'éloignement de l'acteur environnemental. D'où l'intérêt d'envisager de façon globale l'intégration

8. Ce sont les psychologues qui ont le mieux montré les effets rassurants de la proximité en s'intéressant, par exemple, aux effets du contact tactile sur des malades. À ce sujet, Gueguen (2004, p. 232-234) cite les études menées sur les effets du contact d'une infirmière sur les patients. Ces études montrent un effet de réduction de l'anxiété (travaux de Whitcher et Fisher, de Weiss) et un effet d'apaisement émotionnel (travaux de Fisher et Joseph). 
de cette incertitude par acteurs dans le fonctionnement des PME. D'autant plus que lorsque les acteurs proches sont envisagés comme moins incertains, la performance de la PME est meilleure.

Il semble donc que le «proxemicing behavior» ait une portée beaucoup plus générale. Non seulement de nombreuses décisions en PME semblent fortement sensibles à ce principe proxémique, comme nous l'avons montré précédemment (section 2), mais notre étude indique que les perceptions du dirigeant sont également sensibles à ce phénomène.

Nous voudrions conclure en incitant les «PMistes » à réaliser des études empiriques dans d'autres domaines. Une piste novatrice à explorer serait de distinguer les effets positifs et négatifs de la proximité. À ce jour, les preuves empiriques sont encore maigres d'autant plus que notre contribution avait une volonté exploratoire. Si la proximité présente de multiples avantages, elle peut aussi engendrer des effets négatifs. Par exemple, l'exacerbation de l'effet de proximité peut amener le dirigeant à occulter totalement ce que Julien appelle les signaux faibles. «Les signaux faibles sont ceux que généralement les gens d'affaires perçoivent mal, soit parce qu'ils s'y intéressent peu, soit à cause d'un langage, d'objectifs et de formes organisationnelles trop éloignées de leurs préoccupations ou de leurs façons d'appréhender la réalité» (Julien, 2001, p. 149). Or, ces signaux faibles sont souvent à l'origine de l'innovation et de la croissance forte et rapide des entreprises de faible taille. La petitesse des entreprises accentue la domestication des pratiques de gestion et la constitution d'un «chez-soi», lieu privé par excellence où le dirigeant exerce pleinement son emprise, ce qui rend possible une gestion personnalisée. Cette domestication permet également d'expliquer l'allergie de certains dirigeants de petites entreprises à l'égard de l'État.

\section{Bibliographie}

AKerlof, G. (1970), «The market for lemons: quality uncertainty and the market mechanism », Quarterly Journal of Economics, vol. 84.

BAH,T. (2002), «Le marché de la transmission d'entreprise en France: constats et généralités », Colloque «Les enjeux de la transmission d'entreprise », Montpellier, 5-6 décembre, p. 10-24.

Belletante, B. (1991), «Pour une approche des spécificités financières de la PME au travers du concept de territoire financier», Revue internationale PME, vol. 4, $\mathrm{n}^{\mathrm{o}} 1$, p. 30-49. 
Binks, M.R. et C.T. EnNew (1997), «Smaller businesses and relationship banking: the impact of participative behavior», Entrepreneurship, Theory and Practice, vol. 21, n 4, été, p. 83-92.

Bridge, S., K. O’Neill et S. CRomie (1998), Understanding Enterprise, Entrepreneurship and Small Business, Basingstoke, Macmillan Press.

Chandler, G.N. et S.H. HANKs (1993), «Measuring the performance of emerging business: a validation study», Journal of Business Venturing, vol. 8, $\mathrm{n}^{\circ} 5$, p. 391-209.

Chassard, Y. et C. Bouruet (2003), «Gestion du personnel et de l'emploi dans les petites entreprises», Regards sur les $P M E, \mathrm{n}^{\circ} 3$.

Churchill, N. et V. Lewis (1983), «Les cinq stades de l'évolution d'une PME», Harvard l'Expansion, p. 51-63.

Courrent, J.M. et O. Torrès (2005), «A proxemic approach of small business: the case of business ethics», 50th International Council of Small Business (ICSB), Washington.

Crevoisier, O. (1997), «Financing regional endogeneous development: the role of proximity capital in the age of globalization», European Planning Studies, vol. 5, no 3, p. 407-415.

Curran, J. et R.A. Blackburn (2001), Researching the Small Enterprise, Londres, Sage publications.

D’Amboise, G. (1993), «Empirical research on SME'S : the past ten years in Canada», Journal of Small Business \& Entrepreneurship, vol. 10, n 2, p. 2-12.

Demailly, A. (2004), Herbert Simon et les sciences de conception, Paris, L'Harmattan, $244 \mathrm{p}$.

Demsetz, H. (1998), L'économie de la firme. Sept commentaires critiques, Colombelles, Éditions Management et Société (EMS).

Dess, G.G. et R.B. Robinson (1984), «Measuring organizational performance in the absence of objective measures: the case of the privately-held firm and conglomerate business unit», Strategic Management Journal, vol. 5, nº 3, p. 265-274.

DunCAN, R.B. (1972), «Characteristics of organizational environments and perceived environmental uncertainty», Administrative Science Quarterly, vol. 17, p. 313-327.

Gervais, M. (1978), «Pour une théorie de l'organisation-PME», Revue française de gestion, $\mathrm{n}^{\circ} 15$, p. 37-48.

GiBв, A.A. (1988), «Towards the building of entrepreneurial models of support for small business », 11th National Small Firms Policy and Research Conference, Cardiff, novembre.

Godener, A. (1994), «Entreprises traditionnelles et entreprises de haute technologie, des chemins de croissance différents? », Annales du management, XII ${ }^{\text {es Journées }}$ nationales des IAE, tome 2, p. 175-192.

Revue internationale P.M.E., vol. 21, n 1, 2008 
Godener, A. (1995), «Les changements de dimension de l'entreprise en croissance», Congrès de l'AIMS, Paris, p. 612-631.

Gueguen, N. (2004), Psychologie de la manipulation et de la soumission, Paris, Dunod.

Gumpert, D.E. et D.P. Boyd (1984), «The loneliness of the small-business owner», Harvard Business Review, novembre-décembre, p. 18-24.

HAdDADJ, S. et A. D'ANDRIA (2001), «Mesurer les phénomènes agissant sur la transmission des PME familiales», Revue française de gestion, $\mathrm{n}^{\circ}$ 132, p. 11-22.

Hall, E. (1981), «Proxémique», dans Y. Winkin (dir.), La nouvelle communication, Paris, Le Seuil, 373 p.

Joffre, P. (1994), Comprendre la mondialisation de l'entreprise, Paris, Economica, $111 \mathrm{p}$.

Johanson, J. et J.E. VAhLne (1977), «The internationalization process of the firm: a model of knowledge development and increasing foreign market commitments », Journal of International Business Studies, printemps-été, p. 23-32.

Julien, P.-A. (dir.) (1998), The State of the Art in Small Business and Entrepreneurship, Brookfield, Ashgate, Aldershot.

Julien, P.-A. (2001), «Les PME à forte croissance et la métaphore du jazz. Comment gérer l'improvisation de façon cohérente », Revue internationale PME, vol. 14 , $\mathrm{n}^{\text {os }} 3-4$, p. $129-161$.

Julien, P.-A. et M. Marchesnay (1988), La petite entreprise, Paris, Vuibert.

Letowski, A. (2003), «Les ressources humaines: ce concept a-t-il un sens dans les très petites entreprises?», dans Les ressources humaines sont-elles gérables? Hommage à Pierre Jarniou, Paris, L'Harmattan.

Mahé de Boislandelle, H. (1996), «L'effet de grossissement chez le dirigeant de PME: ses incidences sur le plan du management des hommes et de la GRH», $\mathrm{III}^{\mathrm{e}}$ Congrès international francophone PME (CIFPME), Trois-Rivières, Québec, p. 101-115.

MAHÉRAult, L. (1999), «Comportement financier des entreprises familiales: approche empirique», Économies et Sociétés, série Sciences de Gestion, ${ }^{\text {os }}$ 26-27, p. 247-272.

Marchesnay, M. (1991), «La PME: une gestion spécifique?», Économie rurale, $\mathrm{n}^{\circ} 206$, p. 11-17.

Messeghem, K. (1999), Contribution à la compréhension de l'adoption d'un modèle d'assurance qualité en PME: le cas des industries agro-alimentaires, Thèse en sciences de gestion, Université de Montpellier I, 365 p.

Messeghem, K. (2003), «Strategic entrepreneurship and managerial activities in SME's », International Small Business Journal, vol. 21, n 2, p. 197-212.

Moles, A. (1976), Micropsychologie et vie quotidienne, Paris, Denoël/Gonthier.

Moles, A. et E. Rohmer (1978), Psychologie de l'espace, Tournai, Casterman.

Revue internationale P.M.E., vol. 21, nº 1, 2008

(C) 2008 - Presses de l'Université du Québec

Édifice Le Delta I, 2875, boul. Laurier, bureau 450, Québec, Québec G1V 2M2 - Tél.: (418) 657-4399 - www.puq.ca

Tiré de: Revue internationale P.M.E., vol. 20, $\mathrm{n}^{\circ \mathrm{s}} 3-4$, sous la direction de Louis Raymond • PME2003N Tous droits de reproduction, de traduction et d'adaptation réservés 
Moreau, R. (2006), «La formation des équipes d'entrepreneurs », Revue de l'Entrepreneuriat, vol. $5, \mathrm{n}^{\circ} 2$, p. 55-68.

Moureau, N. et D. Rivaud-Danset (2004), L'incertitude dans les théories économiques, Paris, La Découverte.

Norton, E. (1991), «Capital structure and small growth firms», Journal of Small Business Finance, vol. 1, n 2, p. 161-177.

OSEO-BDPME (2005), La transmission des petites et moyennes entreprises, Direction des études, $64 \mathrm{p}$.

Parent, J. (1978), Les firmes industrielles - croissance des firmes et structures industrielles, Paris, Presses universitaires de France.

Penrose, E.T. (1959), The Theory of the Growth of the Firm, Oxford, Basil Blackwell.

St-Pierre, J. (1999), La gestion financière des PME - Théories et pratiques, Québec, Presses de l'Université du Québec.

St-Pierre, J. et R. Beaudoin (1995), «L'évolution de la structure de financement après un premier appel public à l'épargne: une étude descriptive», Revue internationale PME, vol. 8, $\mathrm{n}^{\text {os }} 3$-4, p. 181-203.

Schwach,V.(1993), «Phénoménologie et proxémique:la méthode d'Abraham Moles», Congrès international de sociologie, Sorbonne.

Simon, H.A. (1947), Administration et processus de décision, Paris, Economica, 1983.

Simon, H.A. (1980), Le nouveau management: les décisions par les ordinateurs, Paris, Economica.

Torrès, O. (dir.) (1998), PME: de nouvelles approches, Paris, Economica.

Torrès, O. (2003), «Petitesse des entreprises et grossissement des effets de proximité», Revue française de gestion, $\mathrm{n}^{\circ}$ 144, mai-juin, p. 119-138.

Torrès, O. et P.-A. Julien (2005), «Specificity and denaturation of small business », International Small Business Journal, vol. 23, p. 355-377.

Revue internationale P.M.E., vol. 21, nº 1, 2008

(C) 2008 - Presses de l'Université du Québec

Édifice Le Delta I, 2875, boul. Laurier, bureau 450, Québec, Québec G1V 2M2 • Tél.: (418) 657-4399 - www.puq.ca 